\title{
Vagal Nerve Stimulation has Robust Effects on Neuropsychiatric Assessment in Resistant Epilepsy: A Clinical Series with Clinical Experiences
}

\author{
Hakan EKMEKCI ${ }^{1}$, Hulagu KAPTAN² \\ ${ }^{1}$ Selcuk University, School of Medicine, Department of Neurology, Konya, Turkey \\ ${ }^{2}$ Dokuz Eylul University, School of Medicine, Department of Neurosurgery, Izmir, Turkey \\ Corresponding author: Hulagu KAPTAN hulagukaptan@yahoo.com
}

\section{ABSTRACT}

AIM: To evaluate the resistant epileptic patients who had vagal nerve stimulation (VNS) devices implanted over five years, and to evaluate the neuropsychological aspects of VNS.

MATERIAL and METHODS: Patients with pharmacoresistant epilepsy (PRE) were followed from 2012 to 2017. Totally seven patients were found eligible for VNS in this period. In the pre-surgical period, patients were neuro-psychiatrically assessed by an independent psychiatric committee for mental status, which included assessment of intelligence quotient (IQ), Wechsler adult intelligence scale-revised (WAIS-R) IQ, Stroop test and mini-mental state examination (MMSE), Hamilton depression scale (HDS) and the Beck anxiety inventory (BAI).

RESULTS: The positive effects of VNS on patients' well-being and neuropsychiatric status may be the most as important as the treatment's cost effectiveness. The patients' quality of life (QoL) was calculated just before and 12th / 24th months after VNS implantation. The pre-VNS scores for Quality of Life in Epilepsy (QoLiE-31-P) ranged from 14-59, and average score was 37.14.The post-VNS scores ranged from 31-72, and the average score was 52.86 .

CONCLUSION: VNS has intense effects on QoL of epileptic patients. The best approach for comparing and evaluating QoL in PRE patients may be the QoLiE-31-P inventory, which can be adapted and used in epilepsy centers to evaluate energy, mood, daily activity, cognition, medication effects, seizure worry and overall QoL.

KEYWORDS: Vagal nerve stimulation, Quality of life, Neuropsychiatric assessment, Pharmacoresistant epilepsy

\section{INTRODUCTION}

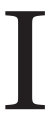
n epilepsy, resistance is said to occur when adequate trials of two tolerated anti-epileptic drugs (AEDs) prescribed according to International League Against Epilepsy (ILAE) guidelines fail to result in complete freedom from seizures(1). This condition of resistance is known as pharmacologically resistant epilepsy (PRE). Resistant seizures can cause cognitive decline and physical injury and are more likely to result in death than curable seizures are. Sudden unexpected death in epilepsy (SUDEP) is the most catastrophic result of resistance. About $30 \%$ of all epileptic patients (including
$20-40 \%$ of patients with primary generalized epilepsy and $60 \%$ of patients with focal epilepsy) may show signs of $\mathrm{PRE}^{2}$. Prolonged, untreated seizures cause neuropsychiatric deficits, impaired cognitive functioning and decreased quality of life (QoL) (2).

Resective surgery may fail if the patient has two or more foci having no localizable epileptogenic zone (EZ) or if the patient experiences seizure onset in a sensitive cortical area $(2,4)$ In the latter case, neuromodulation techniques, including vagal nerve stimulation (VNS), that modify the neuronal synapses, can be useful (3). VNS is a palliative surgery in which a bipolar 
electrode is looped around the left cervical vagal nerve and subsequently connected to an implanted pulse generator, also known as a neuro-cybernetic prosthesis, located beneath the skin on the chest. This device is proposed to control the resistance. The pulse generator is programmed externally to deliver electrical square currency and magnetic pulses of a defined current, pulse width, frequency and delivers square current and magnetic pulses (5). These high-frequency stimulations from the vagal nerve through the brain ultimately inhibit interictal epileptiform discharges.

Although the mechanism of VNS action has not been completely elucidated, it possibly involves diffuse cerebral metabolic changes, cortical and subcortical, through modulation of solitary tract nucleus and brainstem activities ${ }^{6}$. The efficacy of VNS is related to reductions in both the frequency and duration of seizures and to improvements in QoL $(6,7)$.

In epilepsy, the rate of satisfactory, permanent treatment with monotherapy is about $50 \%$. This rate increases by onequarter (25\%) with polytherapy (8). However, roughly $20-30 \%$ of patients remain unresponsive or refractory against all therapies (2). The higher the number of drugs used, the more side effects are observed. AEDs create side effects both in the brain and the systemic vital organs $(9,10)$.

Impaired QoL in epileptic patients with refractory seizures is significantly associated with symptoms of depression, lack of a sense of self-mastery, anxiety, perceived stigma and high seizure frequency. Well-designed studies have found a close association of QoL and psychological distress in refractory epilepsy (11).

Some invasive approaches to treating epilepsy have been developed. Some patients may require cerebral resective surgery. The most commonly encountered type of epilepsy is complex partial epilepsy, in which the possibility of benefiting from invasive procedures is limited(10). The goal of all surgical procedures for epilepsy is to achieve seizure control while preserving memory, learning, speech, temperament, executive functioning and overall QoL (12).

Neuropsychiatric examination is an interdisciplinary approach to diagnostic assessment of mental and brain diseases. When performed skillfully, using a conceptually grounded framework, neuropsychiatric assessment offers the potential for comprehensive diagnostic insight, thereby facilitating optimally informed treatment planning (13).

PRE is related to seizure frequency, co-morbidities, neuropsychological distress and social situation, including self-mastery and perceived stigma. Epilepsy-specific QoL has been quantified using the Quality of Life in Epilepsy (QoLiE-31-P) scale (11), which measures depression, low self-mastery, anxiety, perceived stigma, history of medical and psychiatric co-morbidities and low self-reported medication adherence. It yields precise data on six components related to the patient's neuropsychiatric status: cognition, seizure worry, daily activity, mood, energy and medication effects.

\section{MATERIAL and METHODS}

This study was a clinical trial conducted between June 2012 and November 2017 and included PRE patients who were considered untreatable using conventional and suitable AEDs. All patients were hospitalized at the Selcuk University Education and Research Hospital, in either the Neurology or the Neurosurgery Department. All patients were implanted with VNS devices at the Selcuk University Faculty of Medicine Neurosurgery Department. The Selcuk University Ethics Committee approved the study, issuing it the number 70632468-050.01.04/2017/258. In addition, informed consent was received from each patient or his or her relatives. During the approximately 5.5 years of the study, 7 PRE patients were selected to be followed after having had VNS devices implanted. All patients had clinical PRE, and all were selected by a committee that supervised the VNS device implantations, which included a neurologist, a neurosurgeon and a psychiatrist experienced in epilepsy.

The study evaluated each patient's seizure type, frequency and severity during a minimum of 12 months (maximum: 65, average: 39.3 ) of follow-up using special inventories. At baseline, the study collected patient histories that included demographic data, current AEDs, results of the QoLiE-31-P scale, epilepsy etiology and syndrome, historical seizure types and frequencies and physician global assessment.

All patients had medically refractory epilepsy after having been medically treated for $13.83 \pm 8.22$ years, and all were taking an average of 4 medications at the time of referral. The initial pre-surgical evaluations consisted of clinical neurological exams over multiple years in the Selcuk University Faculty of Medicine Department of Neurology. In addition, cerebral magnetic resonance images (MRIs) and fMRls (1.5 Tesla, Toshiba; Selcuk University Faculty of Medicine, Department of Radiology) were administered. Video electro-encephalograms (EEGs) of the scalp and epilepsy monitoring were conducted long-term at three centres (Gazi University, Ankara; Necmettin Erbakan University; and Selcuk University Faculty of Medicine Department of Neurology, Konya). In the pre-surgical period, patients were neuro-psychiatrically assessed by an independent psychiatric committee for mental status, which included assessment of intelligence quotient (IQ), Stroop test and administration of the mini-mental state examination (MMSE), the Hamilton depression scale (HDS) and the Beck anxiety inventory (BAl) (Table I).

Scalp EEGs were recorded using a digital 19-channel CometPLUS $^{\circledR}$ XL Lab-based EEG (Grass Products, RI, USA) and the TWin ${ }^{\circledR}$ EEG\&LTM Clinical Software. The electrodes were positioned according to the international 10-20 system. Video EEG and epilepsy monitoring were conducted at three abovementioned university centres, but the main collections were conducted at Selcuk University Neurophysiology Lab. and used Neurosoft, Neuron-Spectrum 4/EPM digital 64-channel vEEG (Neuron-Spectrum Video). During the surgery, a VNS device (Cyberonics VNS Therapy System, Model NS Pulse 103, Demipulse Generator and single-pin and subcutaneous implantable VNS apparatus; Houston, TX, USA) was implanted in each patient. In the post-operative period, 


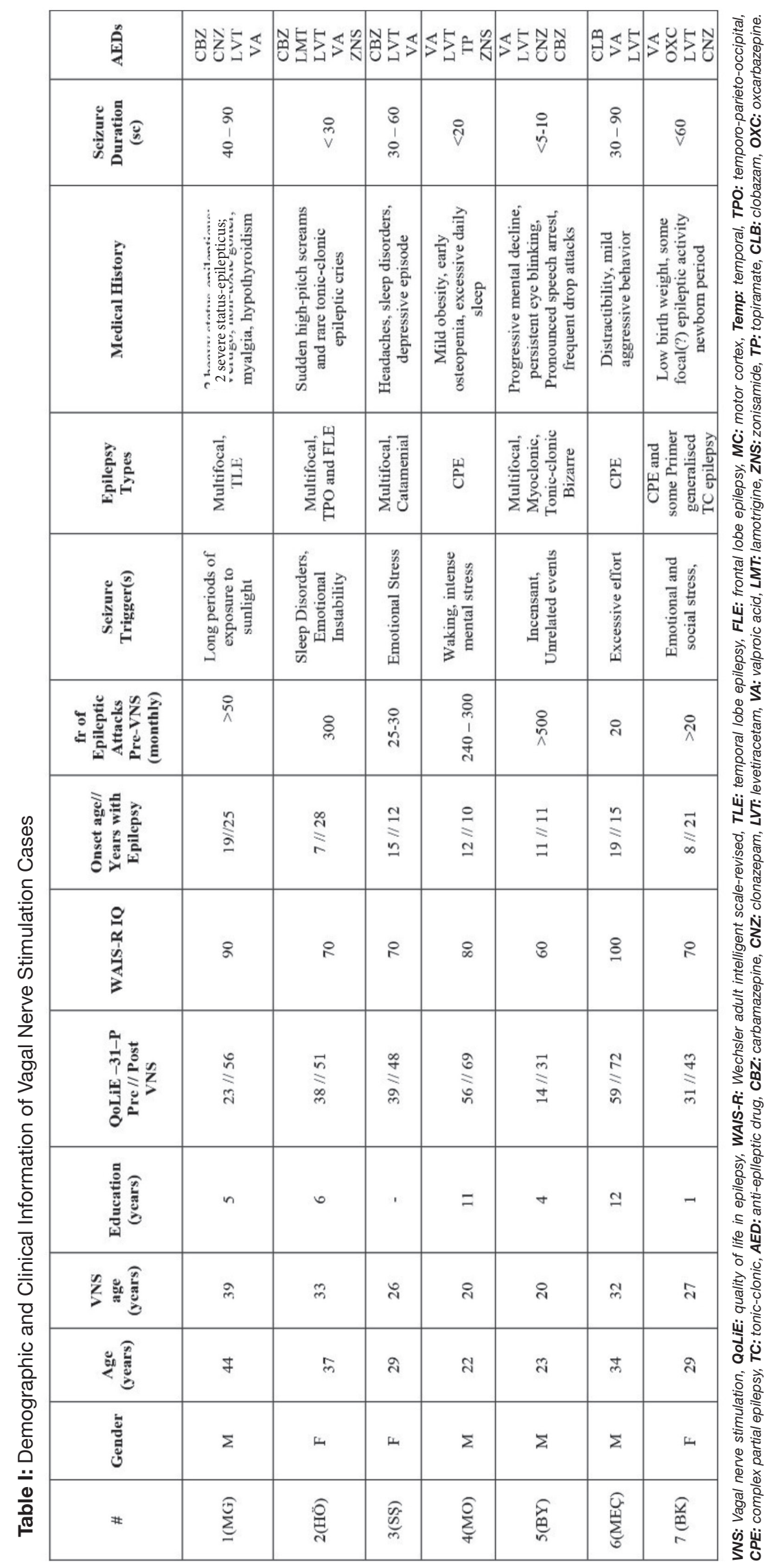


each patient's clinical well-being was evaluated, mainly for seizure improvement, using the Impact on QoL and the scales previously mentioned as having been applied before VNS implantation (Table II).

\section{- RESULTS}

In the present study, 4 male and 3 female patients, whose average age was currently 31.68 years (range: 20-39), were implanted with VNS devices with an average age of 28.14 years at implantation time. All patients had suffered PRE from an early age, 2 of them from age 10. The median age of onset was 13.0 years. The average number of years that the patients had experienced PRE was 17.67.

PRE significantly affects both mental status and education. Although the mandatory length of basic education in Turkey is 12 years, the vast majority of patients in this study had been unable to complete this much education, with only 1 patient graduating from high school and the average number of years of education being only 5.57. The patients' average score on the Wechsler adult intelligence scale-revised (WAIS-R) IQ scale was 77.14. This score indicates borderline deficiency, and we can say these PRE patients had borderline intellectual functioning at the time the VNS devices were implanted. Four of the patients were accepted as being Educable Mentally Retarded or Trainable Mentally Retarded and had attended special-education programmes, but none had been be able to finish the programme at the desired level.

The patients' QoL was calculated just before and again $12^{\text {th }} /$ $24^{\text {th }}$ months after VNS implantation. The pre-VNS scores for QoLiE-31 ranged from 14-59, and average score was 37.14. The post-VNS scores ranged from 31-72, and the average score was 52.86. The increase was about 15.72. Some patients showed better scores at $24^{\text {th }}$ months of post-VNS.

As expected, one of the most significant changes post-VNS was in EEG findings. For example, at 14 months after VNSdevice implantation, Patient 1 showed considerable maturation of background activity and minimal left-hemispheric slowwave activity. Four months before VNS device implantation, his scalp EEG findings had shown localized spikes and sharp waves, highly active epileptiform discharges and increased slow waves with intermittent photonic stimulation (IPS) and hyperventilation (HV).

Brain MRIs, particularly MR-Spectroscopy, were also important to evaluating pre- and post-VNS changes, since not only morphological but also functional findings are involved in VNS-device implantation. Unfortunately, VNS itself is an obstacle to MR imaging. Therefore, the MRIs taken post-VNS were limited. Highly important inferences could be achieved, especially using fMRI, MRI-Spectroscopy, MR angiography and possibly positron emission tomography (PET) and single photon emission computed tomography (SPECT); however, all these are likewise limited mechanically by the presence of a VNS device. In the present study, the MRIs and MRI-Spectroscopies of the patients' brains taken using hydrogen $\left({ }^{1} \mathrm{H}\right)$ showed metabolic changes, mainly in the frontal cortex and the bilateral basal ganglia, and decrement in $\mathrm{N}$-acetylaspar- tate/creatine ratio (NAA/Cr) in the frontal lobes, basal ganglia and subcortical structures.

VNS devices are programmed monthly or in accordance with the tolerance of the patient. The best achievements of VNS are accomplished in about the first six month, during which several neuro-programmings are conducted. The current used for VNS programming ranged from 3-10 mA, and the maximum current output of the magnets ranged from 1.75-3.0 $\mathrm{mA}$. The average number of programming sessions used for programming was 5.7 and the average output currency of the magnets was $2.14 \mathrm{~mA}$. The stimulation frequency ranged from 20-30 Hz, and the average frequency was $28.15 \mathrm{~Hz}$. The other parameter in VNS programming, pulse width, ranged from $130-500 \mathrm{msc}$, and the average was $144.29 \mathrm{msc}$.

Patient 1 (MG), a 39-year old male, tolerated the calibration sessions quite well, and at 60 months post-implantation, his convulsions had decreased from daily to a maximum of 2-4 seizures per month. However, his psychological and physical status improvements were the most impressive, considering that for the last five years, he had been unable to leave his home, had sustained several somatic injuries and had experienced a depressed mood and mild cognitive impairments. In contrast, 60 months after the VNS device implantation, he was able to leave his home for short trips, and his IQ scores had increased at least 10 points at each yearly examination. Both his QoL and seizure control were considerably improved, and he had returned to work part time. Finally, both his HDS and BAS scores showed remarkable improvements.

Patient $2(\mathrm{HO})$, a 37-year old female, had experienced her first seizures at age 8 months. Then, the seizures abated until age seven, when she had one that caused a three-day loss of consciousness. At 20, she experienced a general epileptic attack that yielded myoclonic seizures accompanied by sudden, high-pitched cries. The main foci for the epileptic zone were the temporal and frontal areas. Before VNS implantation, he was being treated with five AEDs. Between 18 months preVNS and 24 months post-VNS, this patient showed a $70.56 \%$ reduction in the maximum number of spikes and a $92.97 \%$ reduction in the number of seizures detected by EEG. PostVNS, her seizures decreased markedly. In addition to showing good EEG stabilization, the VNS procedure reduced her anxiety. Her WAIS-R IQ score of 70 was unchanged after VNS device implantation.

Patient 3 (SS), a 29-year old female, had a seizure history of more than 12 years. She had been diagnosed with secondary generalized, complex partial seizures and tonicclonic attacks and was being treated with three AEDs. She showed occasional symptoms of catamenial C1 epilepsy and generalized, synchronized epileptiform activity. At four months pre-VNS, her slow-wave activity increased with $\mathrm{HV}$, and at three months pre-VNS, with HV and IPS. During the first 6 months post-VNS, her daily seizure frequency decreased from more than 20-30 myoclonic attacks to a few. In the second and third six-month intervals post-VNS, she made some incremental improvements in QoL. She is underdeveloped socially and educationally, as she never attended school, but since VNS implantation, the range of tasks she can perform has increased somewhat. 


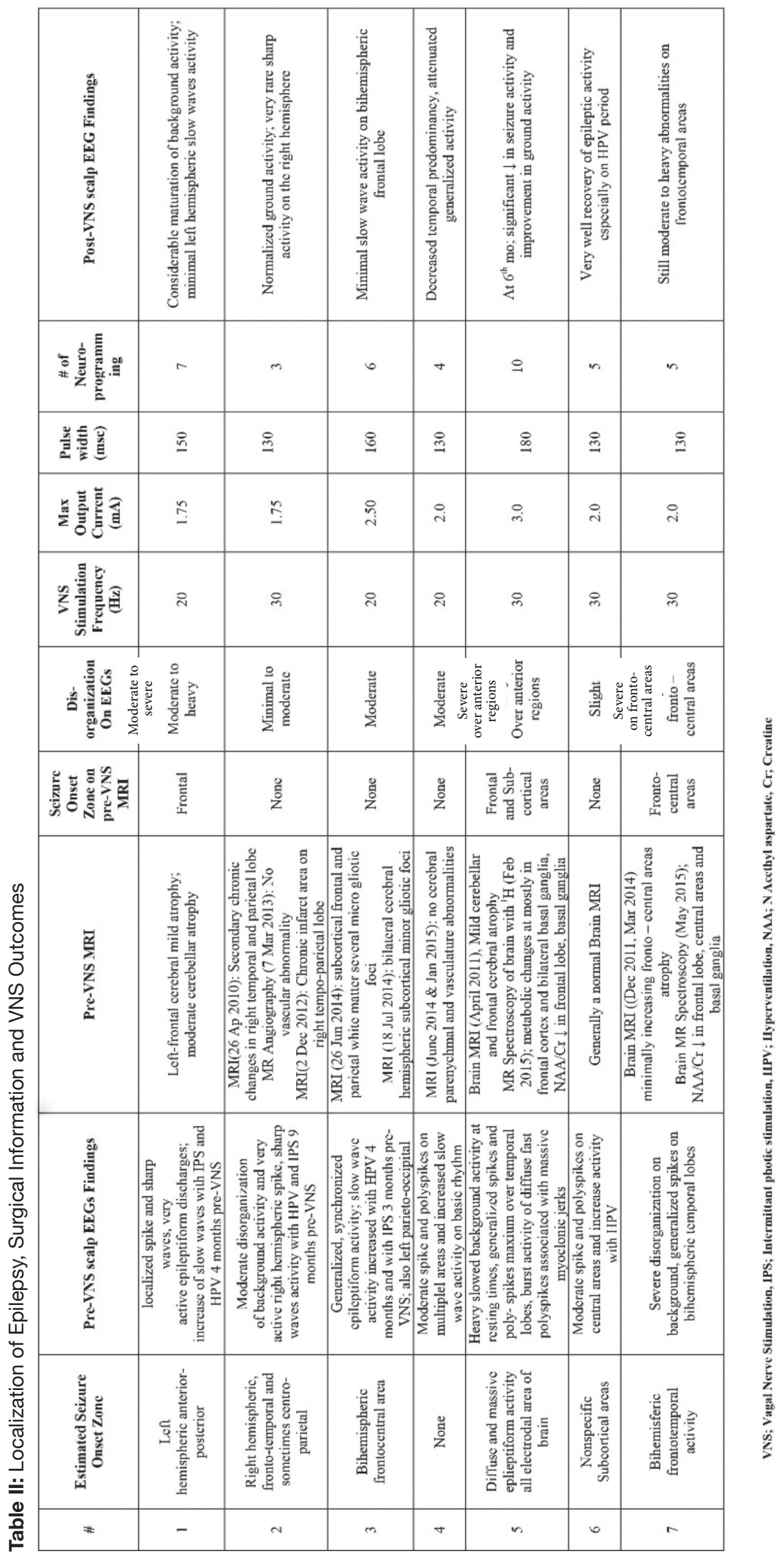


Patient 4 (MO), a 22-year old male, had suffered complex partial (CP) epileptic activity since age 12 . For the last 1.5 years preVNS, he had experienced as many as $12-15$ seizures daily. The most propagative factors in his seizures were intense mental stress in school and life and stressful activity upon waking in the mornings. He was mildly obese, had moderate osteopenia and slept excessively. Post-VNS, his response was dramatic from the beginning and included a seizure-free day within a few weeks. He rejoined school activities to some extent. His sleep disorder was diminished. With a planned diet, he reduced his weight by more than $18 \mathrm{~kg}$. The number of AEDs with which he was treated was reduced from four to two. He achieved good scores in cognition. His BAl score went down markedly. Not only did his mental capacity improve but also his emotional state.

Patient 5 (BY), a 23-year old male, had highly resistant epilepsy and a diagnosis of Lafora Disease (LD), which had been confirmed both genetically and by biopsy. His seizures were categorized as Progressive Myoclonic Epilepsy (PME). He first began experiencing seizures at age 11, and their number increased consistently. In 2013, a biopsy of the eccrine glands was positive for Lafora bodies. In 2014, genetic analysis ${ }^{14}$ showed an EPM2B (malin) genetic mutation at the locus of 6p22-32. His total education was four years of primary school, after which he was unable to continue with school. He suffered very severe, complex and intractable seizures and was being treated with five AEDs, yet his seizure control was very poor. He showed progressive mental decline, persistent eye blinking, pronounced speech arrest, difficulty selecting words and frequent drop attacks. His neuropsychiatric condition was consistent with his rapidly progressing dementia with apraxia. His speech was very idiosyncratic, as word finding, articulations and silent periods in between word production were asynchronous in his clauses. He underwent VNS implantation in April 2015. One year post-VNS, his seizure control had improved, and the number of myoclonic seizures he experienced had declined nearly $40-50 \%$. His most CP seizures nearly disappeared. In the second year after VNS implantation, the number of seizures he experienced began to increase moderately but his excessive salivation and myoclonic episodes remained diminished and his cognitive gains remained. The number of AEDs with which he was treated decreased to four, and the doses of these were being decreased gradually. His Stroop test and QoL scores remained very good. Pre-VNS, his WAIS-R $I Q$ score had been 60 , and post-VNS, it remained the same, but his awareness and responsivity in terms of both speech and tasks clearly improved. These changes have also affected the patient's neuropsychiatric status. He has progressed from being bed-bound to walking, although with extreme difficulty.. The prognosis for LD is very poor, so it is unexpected for a patient to live so long. All other LD patients are known to have died within 10 years of diagnosis. His continued survival of 12 years may be due in part to his implantation with a VNS device. A literature review found that he is only the second LD patient to be implanted with a VNS device. He may be the only case of VNS implantation resulting in semi-controlled PRE and slight improvements in mental and neuropsychiatric statuses after more than 10 years of LD.
Patient 6 (MEC), a 34-year old male, is an agricultural technician who has suffered from resistant CP epilepsy for the last 15 years. Pre-VNS, he was treated with four AEDs, one of which was clobazam 60 mg daily. In 2015, he was implanted with a VNS device, to which he responded dramatically. He returned to his job part time 18 months after surgery. His QoL scores improved, and his emotional healing is evident. In particular, his distractibility and mild aggression, long part of his behaviour, stabilised after VNS implantation.

Patients 7 (BK), a 29-year old female, was a premature baby and had a low birth weight. Her early newborn period was complicated. Her seizures began at age 8 and she was unable to continue her education after that age. Eventually, a mixture of tonic clonic epileptic activity and temporallobe involvement affected her cognition, mental state and neuropsychiatric status. Her EEG findings included mainly prolonged epileptiform activity in the postictal period, and her cerebral MRI findings showed minimal cortical atrophy and diffuse cerebral bihemispheric cortical-subcortical tiny gliotic foci. Her WAIS-R IQ score was 70. She had some eye-contact problems; some addictive behaviours, including to her mother; and slightly limited richness in her speech capacity. Post-VNS, her status began to change in general. In particular, her seizure control improved by more than $50 \%$ and neuropsychiatric problems. Her self-esteem increased somewhat, as did her eye contact, and her daily capacity for speech was enriched. Her BAS score improved moderately, but her pre-VNS benchmark was unreliable, as she was under the strong influence of seizure activities.

Of the seven patients in the present study, two (Patients 1 and 6) of them have been married, and each has two healthy children. No patient has an epileptic person among firstdegree family members, although Patients 5 and 7 have nephews with epilepsy, which is well treated by AEDs. Five patients had WAIS-R IQ scores at about or a little below the value for threshold of low - cut(i.e. 70-80). However, patients 1 and 6 had IQ scores above 90. All patients had experienced the onset of epilepsy as children or teenagers. The most outstanding demographic feature in the study population is the diagnosis of Patient 5 with LD, severe, fatal epilepsy, and the fact that his VNS implantation may be helping to prolong his lifespan with this disease.

\section{DISCUSSION}

This study aimed to identify the quantitative and qualitative factors accounting for clinical, neuropsychiatric and socioemotional improvements in seven selected patients implanted with VNS devices. To that end, it evaluated their pre- and post-surgical EEG recordings, video-EEGs, brain MRIs, MRISpectroscopies and collected data about the time-space orientation of their brains under the effects of VNS stimulation. The study also compared patients' responses based on their types of epilepsy and changes in neuropsychiatric status.

Epilepsy is a nervous-system disease in which mental and somatic health statuses are affected in resistant cases. New treatments include drug therapies and organic involvement 
with the brain. VNS is an exclusive remedy for resistant epilepsy. The vagus nerve is known to be an unrelated structure for epilepsy. The vagal network is involved with the viscera (i.e. the cervical area and the intra-thoracic and intraabdominal organs) and connects a vast area with the brain stem, thalamus and cortical areas (12). The stimulation of the vagus nerve has been found to control epilepsy in animal models in the last 30 years in human beings in the last 20 (12-15). During stimulation, some variables, including current intensity and frequency, pulse width and duration of the 'on' and 'off' periods, can be controlled to tailor the stimulation delivered to the patient (12).

The evidence-based guidelines for VNS accept that depression is a common co-morbidity for people with epilepsy. VNS may provide an additional benefit to those with epilepsy by improving mood. In accordance with the American Academy of Neurology (AAN), VNS may be indicated for improving the mood of adults with epilepsy and may be considered to have improved efficacy over time (Level C) (16). But this potential is attributed as secondary to VNS implantation. The evidence is accepted as being unclear regarding an independent effect on mood in this complex population (Level C).

VNS may be considered progressively effective in patients over multiple years of exposure (Level C). Optimal VNS settings are still unknown, and the evidence is insufficient to support the use of stimulation in either standard or rapid cycling for duty cycle (Level U). VNS may also be considered as an option after a child with PRE has been declared a poor surgical candidate or has had unsuccessful surgery.

In the present study, each patient had a different response to VNS implantation. Indeed, the exact mechanism by which VNS exerts its anti-epileptic effect has not yet been completely elucidated (6). Anatomically, the vagus nerve comprises three types of fibres: $A, B$ and $C$. EEG desynchronization with epileptic activity is abolished mainly by $C$ fibres rather than " $A$ " and "B" fibres (6). VNS influences the locus coeruleus and raphe nuclei to modulate cortical activity by altering noradrenergic and serotonergic projections $(6,17)$. The augmentation of locus coeruleus activity after electrical stimulation of the vagus nerve, demonstrated by an increase in c-fos, may provoke the release of noradrenaline in the limbic circuit and the activation of the dorsal raphe nucleus, which sends diffuse serotonergic projections to the diencephalon and telencephalon (6). In the present study, Patient 1 experienced rapid, significant seizure reduction, which could be related to the working principles of neuronal stimulation on the vagal nerve through the limbic system and thalamic projections. According to the treatment guidelines and classifications of the ILAE, Patient 1 was in "very severe classification", as he suffered from Status Epilepticus (SE) three times and had a very poor response to AEDs. In addition, Patient 5 was known to have LD, which is fatal, although he stays live more than 10 years and nearly three years after VNS implantation. It is unclear whether this patient's life is being prolonged in part by VNS or whether it plays no part in his longevity. Both Patient 1 and Patient 5 responded very well to VNS, being moderate-to-high (> 50\%) in their percentages of seizure reduction during one period.
However, the cognitive effects of VNS have not been fully understood. It is more likely that the effect of VNS on reticular formation could be much more effective for improved cognitive functioning. VNS affects the brain's electrical activity not indirectly through the efferent pathways, but rather directly through the afferent pathways. While the role of VNS on epilepsy has been related to initial increases in extracellular norepinephrine, it has also been shown that VNS increases the levels of free GABA in the cerebrospinal fluid $(6,18,19)$. It can be said of the vagus nerve that physiologically it is the widest part of the autonomous nervous system. It plays an important role in regulating metabolic homeostasis. It has a stabilizing role on the neuroendocrine/immunologic axis via the afferent and efferent pathways $(6,7)$. These complex functions may be related to neuropsychological networks on the limbic system. Thanks to these roles, the number of AEDs used and, more importantly, the adverse effects related to the polypharmacy of patients can be reduced by VNS application $(6,19,20)$

In the present study, Patient 1 gained an average of 33 points on his QoLiE-31-P score (11). This gain was not constant over all testing, but ranged from 12-39 points. His greatest gains were related to energy and daily activity. He made moderate gains in mood and cognition. His smallest improvements were in seizure worry and medication effects. His overall QoL score on the QoliE-31-P increased from 35 to 62.

In contrast, Patients 2 and 3 have not yet displayed very significant changes in cognition since beginning their VNS therapy. However, significant emotional changes have been observed in each. The time interval since their surgeries (about 2.5 years) has not been long enough to provide a definite assessment of the improvement in both their mental and emotional states. However, a positive clinical improvement in the emotional level of Patient 2 was identified two months after her VNS activation, when her myoclonic-based epileptic cries decreased. This patient was a Caucasian woman, and her emotional anxiety prior to the implantation could have been due partly to social and cultural anxiety over not having been married at a young age. Four months post-VNS, an 8-point recovery in her BAl score was observed. Her QoliE31-P score showed an increase of 13 points, with the highest increases being in mood and then energy. Improvement in her neuropsychiatric effects were limited for cognition but were significant for mood and seizure worry.

Patient 3 had catamenial epilepsy and PRE, and her VNS magnet activation may be associated with seizure abortion when used at the time of seizure auras (16) (Level C). This patient had the smallest increase in QoLiE-31-P scores of all patients; her average score increase was 9 . The largest increases were in the mood and seizure worry components. She achieved no increases in cognition and daily activity scores on the QoliE-31-P.

In contrast, Patient 4 , like Patient 6 , had q post-VNS score increase of 13 points on the QoliE-31-P. Patient 4 had had the co-morbidities of obesity, osteopenia and sleep disorder. The obesity may have been related to his high dose of valproic acid. His largest score increases on the QoLiE-31-P were in seizure worry and cognition. He returned to high school 
and had moderate success. Good support on diet and endocrinological treatment may also have helped to increase his seizure control. He reduced his weight to an acceptable body mass index (BMI), and his bony mineralizations were reconstructed.

Patient 5 had very important results. He was diagnosed of LD and is the sole surviving LD patient. His baseline QoliE31-P scores were very low. However, nearly three years postVNS, he had increased his score by 17 points. Among his QoL scores, energy increased the least, but the most robust increase was in cognition, consistent with his progressive dementia, and his daily activity scores increased somewhat. The effects on his mood could not be evaluated. Changes in his neuropsychiatric state were supported by improvements in apraxia, ataxia and neurological speech disorders. This patient showed improvement not only in his progressive mental decline, but also in his persistent eye blinking and pronounced speech arrest, and his frequent drop attacks lessened to some extent.

Ergene et al., after assessing the QoL of VNS patients for one year using the QoLIE-10, found that all scores improved significantly, regardless of seizure reduction $(p<0.01)(4,20)$. Therefore, VNS may positively affect QoL directly, apart from its effect on controlling seizures. Similar results were obtained in a cohort study that included a large number of patients with implanted VNS devices. Responsive and unresponsive patients alike experienced notably improved QoL scores after three months of follow-up $(p<0.0015$ and $p<0.005$, respectively), with there being no statistical difference between the two groups $(4,6,20)$

\section{CONCLUSIONS}

Studies have demonstrated that VNS is an effective therapy for medically refractory partial-onset seizures and primary generalized seizures in patients older than 12 years, yielding an approximate long-term decrease in mean seizure frequency of $40-50 \%$ and a short-term decrease of $20-30 \%$. The present study's early findings of VNS achievement showed that seizure control increased up to $70 \%$ and that patients experienced not only seizure-free periods but also acceptable improvement in daily QoL. The favourable cost-benefit ratio of VNS and its positive effects on patients' well-being and neuropsychiatric statuses indicate that VNS may be an important improvement in the lives of some epileptic patients. VNS is a treatment for resistant epilepsy, but its results seem not to be limited to seizure cessation and epilepsy control. Instead, VNS seems also to provide some very beneficial effects on mental and neuropsychiatric health status. To evaluate the quantitative effects of VNS on the QoL of epilepsy patients with PRE, we applied the QoLiE inventory, specifically the QoLiE-31-P, to measure changes in energy, mood, daily activity, cognition, medication effects, seizure worry and overall QoL.

This study was limited in that it involved a small number of patients; the results of this study indicated that VNS may have important positive effects on the neuropsychiatric aspects of severe epilepsy. This important issue deserves to be investigated in detail.

\section{REFERENCES}

1. Bazil CW, Morrell MJ, Pedley TA: Epilepsy. In: Rowland LP (ed), Merritt's Neurology, 11th ed. Philadelphia: Lippincott Williams \& Wilkins, 2005:990-1008

2. Cramer JA: Exploration of changes in health-related quality of life after 3 months of vagus nerve stimulation. Epilepsy Behav 2(5):460-465,2001

3. Ekmekci $\mathrm{H}$, Kaptan $\mathrm{H}$ : Vagal nerve stimulation. Macedon $\mathrm{J}$ of Med Sci 5(3):391-394,2017

4. Ergene E, Behr PK, Shih JJ: Quality of life assessment in patients treated with vagus nerve stimulation. Epilepsy Behav 2(3):284-287,2001

5. Goldenberg MM: Overview of drugs used for epilepsy and seizures: Etiology, diagnosis, and treatment. Pharmacy and Theurapeutics 35(7):392-415,2010

6. Goldstein MA, Silverman ME: Neuropsychiatric assessment. Psychiatr Clin North Am 28(3):507-547,2005

7. Groves DA, Brown VJ: Vagal nerve stimulation: A review of its applications and potential mechanisms that mediate its clinical effects. Neuroscience and Bio behavioral Reviews 29: 493-500,2005

8. Henry TR. Therapeutic mechanisms of vagus nerve stimulation. Neurology 59(6 Suppl 4):S3-14,2002

9. Janszky J, Hoppe M, Behne F, I Tuxhorn, H, W Pannek, A Ebner: Vagus nerve stimulation: Predictors of seizure freedom. J Neurol Neurosurg Psychiatry 76(3):384-389,2005

10. Krist V, Robrecht R, Naulaerts J, et al: Vagus nerve stimulation. 25 years later! What do we know about the effects on cognition? Neuroscience \& Biobehavioral Reviews 45:6371,2014

11. Kwan P, Arzimanoglou A, Berg AT, De Vogelaere F, Thiery E, Van Roost D, Aldenkamp B, Miatton M, Boon P: Definition of drug resistant epilepsy: Consensus proposal by the ad hoc Task Force of the ILAE Commission on Therapeutic Strategies. Epilepsia 51(6):1069-1077,2010

12. McNamara JO: Drugs effective in the therapy of the epilepsies. In: Hardman JG, Limbird LE, Molinoff PB, Ruddon RW (eds), Goodman \& Gilman's The Pharmacological Basis of Therapeutics, 9th ed. New York: McGraw-Hill, 1999:461-486

13. Morris GL III, Gloss D, Buchhalter J, Mack KJ, Nickels K, Harden C: Evidence-based guideline update: Vagus nerve stimulation for the treatment of epilepsy: Report of the Guideline Development Subcommittee of the American Academy of Neurology. Neurology 81(16):1453-1459,2013

14. Oliveira TVHF, Francisco AN, Junior ZD, Stebel SL: The role of vagus nerve stimulation in refractory epilepsy. Arquivos de Neuro - Psiquiatria 75(9):657-666,2017

15. Penry JK, Dean JC: Prevention of intractable partial seizures by intermittent vagal stimulation in humans: Preliminary results. Epilepsia 31(2):40-43,1990

16. Ridsdale L, Wojewodka G, Robinson E, Landau S, Noble A, Taylor S, Richardson M, Baker G, Goldstein LH and SMILE Team. Characteristics associated with quality of life among People with drug-resistant epilepsy. J Neuro 264:11741184,2017 
17. Scheffer IE, Berkovic SF, Capovilla G, Connolly MB, French J, Guilhoto L, Hirsch E, Jain S, Mathern GW, Moshé SL, Nordli DR, Perucca E, Tomson T, Wiebe S, Zhang YH, Zuberi SM. ILAE classification of the epilepsies: Position paper of the ILAE Commission for Classification and Terminology. Epilepsia 58(4):512-521,2017

18. Turnbull J, Tiberia E, Striano P, Genton P, Carpenter S, Ackerley CA, Minassian BA: Lafora disease. Epileptic Disord 18 Suppl 2:38-62,2016
19. Vera CT, Amorim R, Silvado C, Oliveira AJ, Jorge CL, Faveret E, Ragazzo P, De Paola L: Vagus nerve stimulator in patients with epilepsy: Indications and recommendations for use. Arquivos de Neuro - Psiquiatria 71(11):902-906,2013

20. Wang H, Xiaoguang C, Zhiguo L, Shao Z, Sun B, Shen H, Liu $\mathrm{L}$ : Long-term effect of vagus nerve stimulation on interictal epileptiform discharges in refractory epilepsy. Journal of the Neurological Sciences 284(1):96-102,2009 